\title{
Tapetes de Descanso e Fatores de Risco Laborais- qual a Evidência?
}

rpso.pt/tapetes-de-descanso-e-fatores-de-risco-laborais-qual-a-evidencia/

Santos M, Almeida A, Lopes C, Oliveira T. Tapetes de Descanso e Fatores de Risco Laborais- qual a Evidência? Revista Portuguesa de Saúde Ocupacional on line. 2019, volume 7, 1-?. DOI:10.31252/RPSO.08.06.2019

\section{ANTI-FATIGUE MATS AND LABOR RISK FACTORS - WHAT IS THE EVIDENCE?}

TIPO DE ARTIGO: Revisão Bibliográfica Integrativa/ Scoping Review

Autores: Santos M(1), Almeida A(2), Lopes C(3), Oliveira T(4).

\section{RESUMO \\ Introdução/ enquadramento/ objetivos}

Muitos postos de trabalho têm postura de pé mantida e as consequências para o trabalhador e empregador são frequentemente observadas. As equipas de saúde ocupacional são frequentemente questionadas sobre a importância do uso de tapetes de descanso e/ou qual o modelo que se deveria escolher. Com este estudo, pretende-se conhecer quais os benefícios da utilização dos tapetes e respetiva evidência.

\section{Metodologia}

Trata-se de uma Revisão Bibliográfica Integrativa/ Scoping Review, iniciada através de uma pesquisa realizada em abril de 2019 nas bases de dados "CINALH plus with full text, Medline with full text, Database of Abstracts of Reviews of Effects, Cochrane Central Register of Controlled Trials, Cochrane Database of Systematic Reviews, Cochrane Methodology Register, Nursing and Allied Health Collection: comprehensive, MedicLatina, Academic Search Ultimate, Science Direct, Web of Science, SCOPUS e RCAAP”.

\section{Conteúdo}

A postura de pé mantida associa-se genericamente a desconforto/ dor e fadiga, podendo causar alterações musculares nos membros inferiores e, secundariamente, patologia músculo-esquelética (sobretudo na região lombar, perna e pé).

Os sintomas podem ser atenuados caso se consiga aliviar a pressão plantar. As técnicas usadas são o tapete de descanso, calçado com palmilhas amortecedoras, ortóteses ou plataformas inclinadas.

Os tapetes poderão ser diferenciados em função da rigidez, espessura, compressão e material; bem como aditivos, revestimentos e forma geométrica, todos capazes de alterar a eficácia.

Para além disso, eles também podem ter outros efeitos interessantes (como atenuação do ruído, vibrações, desconforto térmico, risco de queda dos trabalhadores e de objetos, proteção dos chãos, retenção de detritos, distribuição de cargas eletrostáticas ou restrição na propagação do fogo). 


\section{Discussão e Conclusões}

Os estudos publicados sobre este tema são escassos e, para além de, por vezes, tentarem quantificar parâmetros subjetivos, as amostras de trabalhadores envolvidas foram geralmente muito pequenas, pelo que as conclusões divulgadas na literatura não são robustas ou facilmente generalizáveis a todos os funcionários com postura de pé mantida.

Ainda assim, parece consensual entre os documentos selecionados que os tapetes atenuam os sintomas associados a esta questão, permitindo melhorias na produtividade, absentismo, satisfação e até sinistralidade.

Contudo, nos poucos artigos que compararam o uso de tapetes versus rotação entre postura de pé mantida e sentada ou intercalada com caminhadas suaves, estas últimas parecem ser na realidade mais eficazes que os anteriores, ainda que estes sejam já uma mais valia para a Saúde Ocupacional.

PALAVRAS/ EXPRESSÕES- CHAVE: tapetes de descanso, tapetes anti-fadiga, lesões músculoesqueléticas, lombalgia, saúde ocupacional e medicina do trabalho.

\section{ABSTRACT}

\section{Introduction / objectives}

Many jobs have standing work position and the consequences are easily observed. Occupational health teams are often asked about the importance of using resting mats and/ or which model to choose. With this study, it is intended to know the benefits of using resting rugs and if your choice is the most effective to prevent occupational hazards.

\section{Methodology}

This is an Integrated Bibliographic Review, framed in a survey conducted in April 2019 in the databases "CINALH plus with full text, Medline with full text, Database of Summaries of Effects Reviews, Cochrane Central Register of Controlled Trials, Cochrane Database of Systematic Reviews, Cochrane Methodology Register, Nursing and Allied Health Collection: Comprehensive, MedicLatina, Final Academic Search, Direct Science, Web of Science, SCOPUS and RCAAP.

\section{Content}

The standing posture remained associatively to the discomfort/ fatigue, causing symptoms and musculoskeletal pathology (mainly in the lumbar region, leg and foot).

Symptoms can be alleviated if you consider to decrease plantar pressure. The techniques used are the rest rugs, shoes with shock insoles, orthoses or sloping platforms.

The carpets were differentiated according to the rigidity, thickness and material; as well as additives, coatings and geometric shape and all can interfere in the function of the mat.

In addition, they may also have other interesting effects (such as noise attenuation, vibration, thermal discomfort, risk of falling workers and objects, floor protection, debris retention, electrostatic charge distribution or fire propagation restriction).

\section{Discussion and Conclusions}

The studies published on this topic are scarce and, in addition they are attempting to quantify subjective parameters; besides that, the samples of workers involved were generally very small, so the conclusions published in the literature are not robust or easily generalizable to all employees with standing posture.

Nevertheless, it seems consensual among the documents selected that the rugs attenuate the symptoms, allowing improvements in productivity, absenteeism, satisfaction and even accidents 
However, in the few articles that compared the use of mats versus rotation between standing and sitting postures or interspersed with soft walks, the latter seem to be actually more effective than the previous ones, although these are already an added value for Occupational Health.

WORDS / KEY EXPRESSIONS: rest rugs, anti-fatigue mats, musculoskeletal injuries, low back pain, occupational health and occupational medicine.

\section{INTRODUÇÃO}

Muitos postos de trabalho têm postura de pé mantida e as consequências para o trabalhador e empregador são frequentemente observadas. Durante a nossa prática clínica já questionaram certamente a nossa opinião relativamente ao uso de tapetes de descanso e/ou qual o modelo que se deveria escolher. Contudo, ao pesquisar o tema em bases de dados/motores de busca conceituados, percebe-se que as publicações são escassas.

\section{METODOLOGIA}

Pergunta protocolar: Qual a evidência associada ao uso de tapetes de descanso para prevenir lesões músculo-esqueléticas (e respetivos sintomas) em trabalhadores com postura de pé mantida?

Em função da metodologia PICo, foram considerados:

-P (population): trabalhadores com postura de pé mantida.

-I (interest): reunir conhecimentos relevantes sobre as consequências da postura de pé mantida e medidas eficazes para atenuar os seus danos

-C (context): saúde ocupacional nas empresas com postos de trabalho que tenham postura de pé mantida.

Foi realizada uma pesquisa em Abril de 2019 nas bases de dados "CINALH plus with full text, Medline with full text, Database of Abstracts of Reviews of Effects, Cochrane Central Register of Controlled Trials, Cochrane Database of Systematic Reviews, Cochrane Methodology Register, Nursing and Allied Health Collection: comprehensive, MedicLatina e Academic Search Ultimate, Science Direct, Web of Science e SCOPUS".

Contudo, como não se encontraram estudos relativos à realidade portuguesa nestas bases de dados indexadas, os autores procuraram trabalhos inseridos no RCAAP (Repositório Científico de Acesso Aberto em Portugal).

No quadro 1 podem ser consultadas as palavras/ expressões-chave utilizadas nas bases de dados. No quadro 2 estão resumidas as caraterísticas metodológicas dos artigos selecionados.

\section{CONTEÚDO}

\section{Postura de pé mantida- onde existe}

Acredita-se que mais de metade dos trabalhadores europeus fica de pé no trabalho pelo menos três quartos do seu horário [1]. Por vezes a postura de pé mantida, em contexto industrial, é preferida porque permite mais liberdade de movimentos [2]; ela é frequente em diversos postos de trabalho, nomeadamente em caixas de supermercados (ou equivalente), cargos fabris [1] [3], inspetores [3] [4]/ técnicos de controlo de qualidade [3], técnicos de montagem [1] [4], lojistas [1] e profissionais de saúde [1] [3] [4] [5] (sobretudo em contexto cirúrgico).

\section{Postura de pé mantida- semiologia}


A postura de pé mantida associa-se genericamente a desconforto [1] [2] [4] [6] e fadiga [1] [2] [6] [7]. Vários artigos realçam a lombalgia [1] [2] [3] [4] [7] [8], dores nos pés [9], edema [2] [10]/estase venosa [1] [4] [9] e alterações no equilíbrio (com eventuais quedas) [7]. O desconforto pode variar com a altura, peso, idade (mais desconforto se menos jovem) e vínculo laboral [2]. Estar de pé pelo menos duas [3] ou quatro [11] horas seguidas associa-se a lombalgia de forma estatisticamente significativa. A lombalgia é um dos sintomas músculo-esqueléticos mais prevalentes: estima-se que entre 60 a $80 \%$ dos indivíduos a experienciem, sobretudo entre a segunda e a quinta décadas de vida e associa-se, na opinião de alguns investigadores, à contração do glúteo médio. Contudo, ainda que se tenha verificado que o uso de tapetes de descanso consiga atenuar as algias, estes não parecem interferir no músculo atrás mencionado. Pensa-se que a dor (genericamente) se justifica pela acumulação de alguns metabólitos musculares [3].

\section{Postura de pé mantida- consequências}

Trabalhos com postura de pé mantida podem causar alterações musculares nos membros inferiores e, secundariamente, patologia músculo-esquelética [1] (sobretudo na região lombar, perna e pé) [2].

Em 2012 metade dos americanos adultos referiu alguns sintomas músculo-esqueléticas, com destaque para a lombalgia e cervicalgia, que justificaram 291 milhões de dias perdidos de trabalho (absentismo). Na Europa estimou-se que a perda de produtividade totalizou cerca de 240 biliões de euros, atingindo cerca de 40 milhões de trabalhadores. Sabe-se que a patologia músculo-esquelética aumenta com o avançar da idade e com determinadas tarefas laborais [9].

As doenças músculo-esqueléticas podem ter implicações económicas muito relevantes: quer a nível de terapêutica [12], sinistralidade laboral [11], absentismo [3] [11] [12], diminuição da produtividade [3] [11] [12] [13], bem como despesas legais [12] e médicas [11] [13].

\section{Algumas técnicas/ conceitos objetivos para tentar avaliar a situação}

Algumas caraterísticas musculares (como a força de contração) são usadas para estimar a fadiga, sobretudo a nível dos membros inferiores e superiores [1]. $O$ controlo da postura é realizado através da intervenção vestibular, visual e somatosensorial [7].

Uma das respostas comportamentais à postura de pé mantida é a alternância de suporte do peso entre os dois pés; tal permite o reabastecimento de fluido sinovial nas cartilagens afetadas [14].

A pressão plantar aumentada altera a compressão muscular, nervosa e óssea, oscilando (com a postura de pé mantida) entre 70 a $140 \mathrm{KPa}$; a partir de 4 a $5 \mathrm{KPa}$ já se excede a pressão capilar. As superfícies mais rígidas aumentam mais a pressão (o expoente máximo será atingido com o pé descalço, numa superfície como betão, por exemplo) ${ }^{[6]}$.

\section{Técnicas usadas para atenuar as consequências da postura de pé mantida}

A postura de pé mantida contribui para desconforto/ dor, que pode ser atenuado caso se consiga aliviar a pressão plantar. As técnicas usadas são o tapete de descanso [3] [4] [8], calçado com palmilhas amortecedoras [3] [8], ortóteses [3] ou plataformas inclinadas [8]; destes, o tapete constitui a opção mais frequente [3] [7]; no entanto, o seu uso, bem como de palmilhas, não apresenta eficácia consensual na literatura. Para além disso, em algumas investigações é também sugerida a escolha de superfícies menos rígidas e intercalar tarefas com postura de pé mantida com períodos de caminhada- os autores de um estudo concluíram que a capacidade muscular estava diminuída nos trabalhadores com postura de pé mantida, mesmo com uso de tapetes, mas preservada naqueles que intercalavam essa posição com alguns períodos de caminhada [1]. 
Ainda que pouco mencionadas, um estudo estimou uma diferença em $59 \%$ na prevalência da lombalgia com o uso de plataformas inclinadas. Contudo, sem diferenças significativas a nível de volume/ espessura muscular das áreas envolvidas [8].

O uso de palmilhas, por sua vez, poderá ser mais adequado que os tapetes, caso o trabalho apresente alguma mobilidade [2], em vez de tarefas totalmente estáticas.

\section{Caraterísticas dos tapetes}

Os tapetes poderão ser diferenciados em função da rigidez [3], espessura [3] [4] [14], compressão [3] e material [3] [4] [14]; bem como aditivos, revestimentos e forma geométrica, todos capazes de alterar a eficácia [4] [14].

Curiosamente, alguns investigadores defendem que tapetes mais suaves (menos rígidos) podem associar-se a mais desconforto [4]; mas a maioria discorda [3]. Outros, por sua vez, acreditam que o ideal será evitar tapetes simultaneamente muito suaves e muito rígidos [4]. Acredita-se que uma superfície menos rígida potencie o conforto, mas, simultaneamente, aumente a instabilidade [14]. O uso de um tapete, globalmente, proporciona mais conforto que permanecer numa superfície mais rígida. Contudo, se o tapete gerar muita instabilidade, poderá ter o efeito oposto. Quanto à compressão, os menos comprimidos parecem ser mais eficazes (por exemplo, três vezes mais, num caso específico avaliado). A elasticidade também aumenta o conforto e atenua a fadiga [3].

\section{Evidência associada aos tapetes de descanso}

Verifica-se que os tapetes diminuem o desconforto associado à postura de pé mantida ainda que o mecanismo exato não esteja totalmente esclarecido [6]. Um estudo reportou que houve um aumento de $44 \%$ na produtividade e diminuição em $23 \%$ do absentismo, havendo também aumento da segurança [9]. Considera-se, assim, que eles promovem, globalmente, a melhoria das condições de trabalho [5].

O facto de a superfície se tornar um pouco mais maleável e instável, obriga a que o pé tenha micromovimentos, que atenuam a estase venosa [11].

Alguns estudos consultados compararam a eficácia entre tipos diferentes de tapete e, ainda que tenham verificado a atenuação do desconforto em todos [4] [14] (após quatro horas de pé) [4], não conseguiram encontrar diferenças significativas entre os diversos modelos [4] [14], através da avaliação subjetiva de desconforto. Não é fácil avaliar algo não objetivo, por vezes não traduzido para um único parâmetro fisiológico específico [4]. Alguns autores também mencionam que, presentemente, pode ser difícil encontrar grandes diferenças entre tapetes porque, eventualmente, ao longo dos anos, os de pior qualidade foram menos vendidos e, entretanto, retirados do mercado [4] [14]. Para além disso, alguns estudos envolvem amostras muito pequenas, pelo que os resultados poderão não espelhar com rigor a realidade.

Um dos artigos salientou como limitação terem sido usadas sessões de quatro horas com postura de pé mantida (que poderão não ser representativas de turnos de oito e doze horas, existentes em alguns setores), para além de que diferentes modelos de sapatos poderão influenciar a avaliação de eficácia dos tapetes, em ambos os sentidos; a suscetibilidade individual à dor também varia muito. Seria desejável, segundo alguns investigadores, usar técnicas de estudo fisio-anatómicas mais rigorosas, para perceber melhor todo o processo [4].

Quantificar atitudes comportamentais, como oscilar o peso corporal entre os dois pés, poderá ser mais representativo do desconforto e assim se perceber qual o modelo mais adequado. Alternar o apoio do peso entre os dois pés alivia temporariamente a pressão o que, por sua vez, permite a circulação de fluido sinovial na cartilagem/ articulação e diminui a estase venosa [4] (através de micro-movimentos dos membros inferiores) [9]. 
A alternância de pés demonstrou-se correlacionada com o desconforto reportado e com a rigidez do piso [4]. Um estudo verificou que a existência de tapetes de descanso aumenta de forma estatisticamente significativa a alternância de peso entre os membros inferiores [12], como adaptação à postura de pé mantida.

Contudo, alguns investigadores salientam que, ainda que os tapetes atenuem os sintomas, mais eficaz é diminuir o número de horas com postura de pé mantida (através da rotação com postos com tarefas sentadas) [4] ou intercalando com períodos de caminhada suave; contudo, existem postos de trabalho onde tal não é possível- nestas situações poderão ser usados os tapetes de descanso [9]. A postura de pé mantida aumenta a pressão intramuscular nos membros inferiores que, por sua vez, pode alterar o fluxo sanguíneo e respetiva oxigenação; essa pressão intramuscular baixa com a caminhada suave, atenuando-se assim a fadiga e o edema local [1]. Intercalar a postura de pé mantida com caminhadas é então mais eficaz que o uso de tapetes [14].

Um dos estudos salientou como limitação o facto de não se conseguir impedir que o trabalhador avaliado saiba se está ou não em cima de um tapete o que, em alguns casos, poderá criar um efeito placebo e enviesar a avaliação. Para além disso, não existirem diferenças entre modelos nos tempos avaliados, não significa que estas não surjam a médio e longo prazos [14].

A não se verificarem grandes diferenças entre os diversos modelos analisados, alguns autores sugerem que a escolha se possa orientar por critérios como custo, durabilidade e segurança [4] [14].

\section{Outras vantagens/ funções que os tapetes de descanso podem assumir}

Para além das questões já mencionadas a nível músculo-esquelético, os tapetes podem ter outras propriedades adicionais pertinentes, como o facto de serem antiderrapantes, resistentes a agentes químicos, retardadores de fogo, dissipadores de cargas eletrostáticas, antimicrobianos [11], atenuadores das vibrações [12] [15], isolantes, protetores da queda de trabalhadores ao mesmo nível e de objetos, bem como por atenuarem o desconforto térmico e ruído [12] ou reterem detritos [9].

Acredita-se que os tapetes consigam atenuar as vibrações, ainda que a potência do efeito pareça depender da composição e formato do dispositivo; alguns defendem que é necessária mais investigação [5]. Contudo, outros investigadores discordam dessa capacidade dos tapetes [10].

\section{DISCUSSÃOI CONCLUSÃO}

Os estudos publicados sobre este tema são escassos e, para além de, por vezes, tentarem quantificar parâmetros subjetivos, as amostras de trabalhadores envolvidas foram geralmente muito pequenas, pelo que as conclusões divulgadas na literatura não são robustas ou facilmente generalizáveis a todos os funcionários com postura de pé mantida.

Ainda assim, parece consensual entre os documentos selecionados que os tapetes atenuam os sintomas associados a esta questão, permitindo melhorias na produtividade, absentismo, satisfação e até sinistralidade. Para além disso, eles também podem ter outros efeitos desejáveis interessantes (como atenuação do ruído, vibrações, desconforto térmico, risco de queda dos trabalhadores e de objetos, proteção dos chãos, retenção de detritos, distribuição de cargas eletrostáticas ou atenuadores de propagação do fogo).

Contudo, nos poucos artigos que comparam o uso de tapetes versus rotação entre postura de pé mantida e sentada ou intercalada com caminhadas suaves, estas últimas parecem ser na realidade mais eficazes que os anteriores, ainda que estes sejam já uma mais valia para a Saúde Ocupacional.

\section{CONFLITOS DE INTERESSE, QUESTÕES ÉTICAS E/OU LEGAIS}

Nada a declarar. 


\section{AGRADECIMENTOS}

Nada a declarar.

\section{BIBLIOGRAFIA}

[1] Garcia M, Steinhilder B, Laudli T, Martin B. Long-lasting changes in muscle twich force during simulated work while standing or walking. Human Factor. 2016, 58(8), 1117-1127. DOI: $10.1177 / 0018720816669444$

[2] Noor S. Ahmad I, Wahab N, Ma'arof M. A review of studies concerning prolonged standing working posture. Advanced Engineering Forum. 2013, 10, 131-136. DOI: 10.4028.www.scientific.net/AEF.10.131

[3] Aghazadech J, Ghaderf M, Azghani M, Khalkhali H, Allahyari T, Mohebbi I. Anti-Fatigue Mats, low back pain and electromyography: an interventional study. International Journal of Occupational Medicine and Environmental Health. 2015, 28(2), 347-356. DOI: 10.13075/jomeb

[4] Wiggermann N, Keyserling W. Effects of Anti-fatigue Mats on perceived disconfort and weight-shifting during prolonged standing. Human Factors. 2013, 55(4), 764-775. DOI: 10.1177/00187200812466672

[5] Taiar R, Chiomentin X, Abdi E, Polidori G, Ahran T. The biomechanics and Ergonomics of the impact of Anti-Fatigue Mats on decreasing whole body vibration. International Conference of Applied Human Factors in Ergonomics. AHFE2017: Advances in Physical Ergonomics and Human Factors. 2017, 6066.

[6] Wiggermann N, Keyserling M, Pokerney B. The effect of flooring characteristics on plantar pressures on the foot during standing. Procedings of the Human Factors and Ergonomic Society. 2010, 20152018.

[7] Soangra R, Lockhart T. Determination of stabilization diffusion analysis coefficients and invariante density analysis parameters to understand postura stability associated with analysis parameters to understand postura stability associated with standing on Anti-Fatigue Mats. Biomedical Sciences Instrumentation. 2012, 48, 415-422.

[8] Gallaghner K, Wong A, Callaghan J. Possible mechanisms for the reduction of low back pain associated with standing on a sloped surface. Gait \& Posture. 2013, 313-318.

[9] O'Connor R. Anti-Fatigue Mats keep workers on their feet? Assembly. 2018, 61(11), 68-69.

[10] Leduc M, Eger T, Godwin A, Dickey J, Oliver M. Evaluation of transmissibility properties of AntiFatigue Mats used by workers exposed to foot-transmitted vibration. Canadian Acoustic. 2011, 39(2), 88-90.

[11] Taiar R. New study confirms benefits of Anti-Fatigue Mats. Ergonoma Journal. 2012, 27(13).

[12] Taiar R, Ahram T, Gardan N, Schneider A, Sifaki- Pistolla D. Impact of advance Fabrics on Biomechanics: exemple of Anti-Fatigue Mats. Advances in Ergonomics Modeling Usability of Special Populations. 2016, 301-313.

[13] Boston J. Fighting Fatigue from the ground up. Occupational Health \& Safety. 2011, 80(7), 88-92.

[14] Wiggerman N, Keyserling M. Effect of flooring on disconfort and behavioral responses to prolonged standing. Procedings of the Human Factors and Ergonomic Society $54^{\text {th }}$ Annual Meeting. 2010, 783787.

[15] Boucher D, Oliver M, Eger T. Quantification and comparison of selected material properties for AntiFatigue Mats to investigate vibration transmission reduction potencial. Canadian Acoustic. 2011, 39(2), 86-87. 


\begin{tabular}{|c|c|c|c|c|c|c|c|}
\hline $\begin{array}{l}\text { Motor de } \\
\text { busca }\end{array}$ & Passwords & Critérios & $\begin{array}{l}N^{0} \text { de } \\
\text { documentos } \\
\text { obtidos }\end{array}$ & $\begin{array}{l}\mathrm{N}^{\circ} \mathrm{da} \\
\text { pesquisa }\end{array}$ & $\begin{array}{l}\text { Pesquisa } \\
\text { efetuada } \\
\text { ou não }\end{array}$ & $\begin{array}{l}\mathrm{N}^{\circ} \text { do } \\
\text { documento } \\
\text { na } \\
\text { pesquisa }\end{array}$ & $\begin{array}{l}\text { Categorização } \\
\text { inicial do } \\
\text { documento }\end{array}$ \\
\hline \multirow{2}{*}{$\begin{array}{l}\text { Academic } \\
\text { Search } \\
\text { Ultimate }\end{array}$} & Rest rugs & \multirow{18}{*}{$\begin{array}{l}-2009 \text { a } \\
2019 \\
\text {-acesso } \\
\text { a } \\
\text { resumo }\end{array}$} & 3 & 1 & $\operatorname{sim}$ & & \\
\hline & $\begin{array}{l}\text { Anti-fatigue } \\
\text { mats }\end{array}$ & & 12 & 2 & $\operatorname{sim}$ & $1-5$ e 10 & 2.1 a 2.6 \\
\hline \multirow{3}{*}{$\begin{array}{l}\text { Science } \\
\text { Direct }\end{array}$} & Rest rugs & & 1504 & 3 & não & & \\
\hline & $\begin{array}{l}\text { Anti-fatigue } \\
\text { mats }\end{array}$ & & 1014 & 4 & não & & \\
\hline & $3+4$ & & 23 & 5 & $\operatorname{sim}$ & & \\
\hline \multirow[t]{13}{*}{ SCOPUS } & Rest rugs & & 2 & 6 & $\operatorname{sim}$ & & \\
\hline & $\begin{array}{l}\text { Anti-fatigue } \\
\text { mats }\end{array}$ & & 22 & 7 & $\operatorname{sim}$ & $\begin{array}{l}1 \\
2\end{array}$ & $\begin{array}{l}7.1 \\
=2.5\end{array}$ \\
\hline & & & & & & 3 & 7.2 \\
\hline & & & & & & 5 & 7.3 \\
\hline & & & & & & 7 & 7.4 \\
\hline & & & & & & 9 & $=2.1$ \\
\hline & & & & & & 11 & $=2.2$ \\
\hline & & & & & & 14 & 7.5 \\
\hline & & & & & & 16 & 7.6 \\
\hline & & & & & & 17 & 7.7 \\
\hline & & & & & & 19 & 7.8 \\
\hline & & & & & & 20 & 7.9 \\
\hline & & & & & & 21 & 7.10 \\
\hline \multirow{7}{*}{$\begin{array}{l}\text { Web of } \\
\text { Science }\end{array}$} & Rest rugs & & 30 & 8 & $\operatorname{sim}$ & & \\
\hline & $\begin{array}{l}\text { Anti-fatigue } \\
\text { mats }\end{array}$ & & 10 & 9 & $\operatorname{sim}$ & $\begin{array}{l}2 \\
3\end{array}$ & $\begin{array}{l}=2.5 \\
=2.3\end{array}$ \\
\hline & & & & & & 5 & $=2.1$ \\
\hline & & & & & & 6 & $=2.2$ \\
\hline & & & & & & 7 & 9.1 \\
\hline & & & & & & 8 & 9.2 \\
\hline & & & & & & 10 & $=7.8$ \\
\hline \multirow[t]{2}{*}{ RCAAP } & $\begin{array}{l}\text { Tapetes de } \\
\text { Descanso }\end{array}$ & & 0 & 10 & não & & \\
\hline & $\begin{array}{l}\text { Tapetes } \\
\text { anti-fadiga }\end{array}$ & & 0 & 11 & não & & \\
\hline EBSCO & Rest rugs & -2009 a & 1 & 12 & $\operatorname{sim}$ & & \\
\hline
\end{tabular}




\begin{tabular}{|c|c|c|c|c|c|c|c|}
\hline & & & & & & & \\
\hline $\begin{array}{l}\text { Database of } \\
\text { Abstracts } \\
\text { and }\end{array}$ & $\begin{array}{l}\text { Anti-fatigue } \\
\text { mats }\end{array}$ & $\begin{array}{l}\text {-acesso } \\
\text { a } \\
\text { resumo }\end{array}$ & 5 & 13 & $\operatorname{sim}$ & $\begin{array}{l}1 \\
2\end{array}$ & $\begin{array}{l}=2.5 \\
=2.1\end{array}$ \\
\hline $\begin{array}{l}\text { Reviews, } \\
\text { Central }\end{array}$ & & & & & & 3 & $=2.2$ \\
\hline $\begin{array}{l}\text { Register of } \\
\text { Controlled }\end{array}$ & & & & & & 4 & $=9.1$ \\
\hline $\begin{array}{l}\text { Trials, } \\
\text { Cochrane } \\
\text { Database of } \\
\text { Systematic } \\
\text { Reviews, } \\
\text { Nursing \& } \\
\text { Allied Health } \\
\text { Collection e } \\
\text { MedicLatina) }\end{array}$ & & & & & & 5 & $=7.8$ \\
\hline
\end{tabular}

Quadro 2: Caraterização metodológica dos artigos selecionados

\section{Artigo Caraterização Resumo metodológica}

$\begin{array}{lll}\text { Estudo } & \text { Este estudo europeu utilizou uma amostra de dez trabalhadores do sexo } \\ \text { experimental } & \text { masculino e oito do sexo feminino; colocou-os em três situações: com postura de } \\ \text { pé mantida numa superfície dura, com um tapete de descanso e num tapete } \\ \text { rolante, por } 320 \text { minutos, intercalados com três momentos sentados. Os autores } \\ \text { concluíram que a caminhada suave potenciava as caraterísticas musculares } \\ \text { avaliadas, mesmo de forma superior à que o uso do tapete proporcionou. }\end{array}$

2 Revisão bibliográfica narrativa

Nesta revisão os autores pretenderam pesquisar quais as principais consequências que a postura de pé mantida pode acarretar, quer a nível de sintomas, quer patologias; deram algum destaque para o uso de tapetes de descanso, como forma de orientar melhor as intervenções ergonómicas. Concluíram que o uso de tapetes e de palmilhas atenuou os sintomas, ainda que mais investigação deva ser concretizada.

$3 \quad$ Estudo experimental randomizado duplamente cego

Este documento iraniano registou uma investigação na qual os autores desenharam um projeto para investigar a dor/ desconforto associados à postura de pé mantida, bem como as alterações verificadas a nível do glúteo médio, musculo esse destacado em relação à lombalgia, salientando o uso de tapetes de descanso. Concluíram que estes dispositivos atenuam os sintomas, ainda que não alterem os parâmetros musculares avaliados.

$4 \quad$ Estudo experimental (distribuídos uniformemente por sexo) foi sujeito a quatro horas de postura de pé mantida, numa superfície dura e em cima de quatro modelos diferentes de tapetes de descanso; foram quantificados os sintomas subjetivos e a pressão no pé. A dor/ desconforto não teve sensibilidade para distinguir os diferentes modelos de tapetes, sendo que a alternância de peso entre os dois pés possa constituir uma avaliação mais objetiva do alívio sintomático, segundo os investigadores em causa.

\section{$5 \quad$ Estudo} experimental

Neste artigo foi usada uma amostra (com características não especificadas) que avaliou a capacidade de sete diferentes modelos de tapetes atenuarem as consequências das vibrações, através de um acelerómetro. Concluiu-se que estes eram eficazes em relação às vibrações, melhorando as condições de trabalho.

$6 \quad$ Estudo piloto

Esta publicação norte-americana pretendeu investigar se a alteração na pressão do pé poderá justificar os sintomas relacionados com a postura de pé mantida, sobretudo quando se usam tapetes anti-fadiga. Percebeu-se que superfícies menos rígidas (por exemplo, através do uso de tapetes ou determinados modelos de calçado) atenuam a pressão no pé. 


$\begin{array}{ll}7 \text { Estudo piloto } & \text { Neste projeto de investigação norte-americano utilizou-se uma amostra de sete } \\ & \text { voluntários (dos quais cinco pertenciam ao sexo masculino) e testou-se a } \\ & \text { estabilidade postural associada à postura de pé mantida, com olhos abertos (com } \\ & \text { e sem tapete) e com olhos fechados, para avaliar a fadiga muscular. Os autores } \\ & \text { concluíram que os resultados em relação aos tapetes, para períodos } \\ & \text { prolongados, não foram tão promissores quanto o esperado, ainda que também } \\ & \text { salientem que mais pesquisa deverá ser realizada. }\end{array}$

8 Estudo piloto Nesta publicação oriunda do Canadá, usou-se uma amostra de onze estudantes universitários (seis dos quais do sexo masculino), convidados a permanecer 16 minutos em postura de pé mantida, em solo plano e piso inclinado (a subir e a descer), respetivamente. Os autores verificaram que a atenuação da lombalgia efetivamente ocorre nas superfícies inclinadas, ainda que tal pareça não se associar às alterações musculares no tronco, mas ao diferente posicionamento dos membros inferiores e oscilações no apoio do peso.

\begin{tabular}{lll}
\hline 9 & $\begin{array}{l}\text { Artigo de } \\
\text { opinião }\end{array}$ & $\begin{array}{l}\text { Este artigo simples realça alguns estatísticas norte-americanas relativas a } \\
\text { doenças músculo-esqueléticas, dias perdidos de trabalho e algias associadas; } \\
\text { bem como produtividade, absentismo e custos relacionados. Menciona-se } \\
\text { também, sumariamente, a forma como se supõe que os tapetes consigam } \\
\text { atenuar os sintomas associados à postura de pé mantida. }\end{array}$ \\
\hline $10 \quad \begin{array}{l}\text { Estudo } \\
\text { experimental }\end{array}$ & $\begin{array}{l}\text { Este documento proveniente do Canadá pretendeu estudar a eventual atenuação } \\
\text { das vibrações através do uso de tapetes de descanso. Foram avaliados três } \\
\text { modelos diferentes e nenhum proporcionou resultados muito encorajadores. }\end{array}$ \\
\hline $\begin{array}{l}\text { Artigo de } \\
\text { opinião }\end{array}$ & $\begin{array}{l}\text { Um dos investigadores que se dedica a esta área publicou um texto simples, no } \\
\text { qual menciona sumariamente as consequências associadas à postura de pé } \\
\text { mantida e a forma como os tapetes podem atuar para atenuar tal, mencionando } \\
\text { ainda outras funções que estes podem ter em contexto laboral (a nível de } \\
\text { quedas, incêndio, cargas eletrostáticas e propriedades antimicrobianas). }\end{array}$ \\
\hline
\end{tabular}

\begin{tabular}{lll}
\hline 12 & $\begin{array}{l}\text { Estudo } \\
\text { transversal } \\
\text { descritivo } \\
\text { correlacional }\end{array}$ & $\begin{array}{l}\text { Nesta investigação utilizou-se uma amostra constituída por 25 elementos (15 dos } \\
\text { quais do sexo masculino), eliminando elementos com patologias que pudessem } \\
\text { alterar o equilíbrio. Os autores verificaram que a alternância de peso/ postura } \\
\text { ajuda a atenuar a pressão no pé. }\end{array}$ \\
\hline 13 & $\begin{array}{l}\text { Artigo de } \\
\text { opinião }\end{array}$ & $\begin{array}{l}\text { Neste texto simples, os autores descrevem algumas condições associadas aos } \\
\text { tapetes, interessantes a nível ocupacional, com realce para produtividade, custos } \\
\text { e sinistralidade. }\end{array}$
\end{tabular}
14 Estudo Este artigo norte-americano utilizou uma amostra de dez estudantes que foi experimental colocada quatro horas de pé em quatro diferentes modelos de tapetes e quatro horas numa superfície rígida sem tapete. Verificaram que todos os tapetes foram mais confortáveis do que sem tapete, ainda que não se tenham encontrado diferenças estatisticamente significativas entre os diversos modelos.

$15 \quad \begin{aligned} & \text { Estudo } \\ & \text { experimental }\end{aligned}$

Esta investigação proveniente do Canadá testou vários tipos de tapetes perante a amortização de vibrações e conclui que tal capacidade varia entre modelos, provavelmente associada à compressão, rigidez e flexibilidade. 
Licenciada em Medicina; Especialista em Medicina Geral e Familiar; Mestre em Ciências do Desporto; Especialista em Medicina do Trabalho e Doutoranda em Segurança e Saúde Ocupacionais, na Faculdade de Engenharia da Universidade do Porto. Presentemente a exercer nas empresas Medicisforma, Servinecra e Securilabor; Diretora Clínica das empresas Quercia e Gliese; Diretora da Revista Portuguesa de Saúde Ocupacional on line. Endereços para correspondência: Rua Agostinho Fernando Oliveira Guedes, 42, 4420-009 Gondomar. E-mail: s_monica_santos@hotmail.com.

\section{(2)Armando Almeida}

Doutorado em Enfermagem; Mestre em Enfermagem Avançada; Especialista em Enfermagem Comunitária; Pós-graduado em Supervisão Clínica e em Sistemas de Informação em Enfermagem; Docente na Universidade Católica Portuguesa, Instituto da Ciências da Saúde - Escola de Enfermagem (Porto); Diretor Adjunto da Revista Portuguesa de Saúde Ocupacional on line. 4420-009 Gondomar. E-mail: aalmeida@porto.ucp.pt.

\section{(므)Catarina Lopes}

Licenciada em Enfermagem, desde 2010, pela Escola Superior de Saúde Vale do Ave. A exercer funções na área da Saúde Ocupacional desde 2011 como Enfermeira do trabalho autorizada pela Direção Geral de Saúde, tendo sido a responsável pela gestão do departamento de Saúde Ocupacional de uma empresa prestadora de serviços externos durante 7 anos. Atualmente acumula funções como Enfermeira de Saúde Ocupacional e exerce como Enfermeira Generalista na SNS24. Encontra-se a frequentar o curso Técnico Superior de Segurança do Trabalho. 4715-028. Braga. Email: catarinafflopes@gmail.com

\section{(4)Tiago Oliveira}

Licenciado em Enfermagem pela Universidade Católica Portuguesa. Frequenta o curso de Técnico Superior de Segurança no Trabalho. Atualmente exerce a tempo inteiro como Enfermeiro do Trabalho. No âmbito desportivo desenvolveu competências no exercício de funções de Coordenador Comercial na empresa Academia Fitness Center, assim como de Enfermeiro pelo clube de futebol União Desportiva Valonguense.4435-718 Baguim do Monte. E-mail: tiago_sc16@hotmail.com. 